\title{
Tunable Resonant-Cavity-Enhanced Photodetector with Double High-Index-Contrast Grating Mirrors
}

\author{
Learkthanakhachon, Supannee; Yvind, Kresten; Chung, II-Sug
}

Published in:

Proceedings of SPIE - The International Society for Optical Engineering

Link to article, DOI:

$10.1117 / 12.2004047$

Publication date:

2013

Link back to DTU Orbit

Citation (APA):

Learkthanakhachon, S., Yvind, K., \& Chung, I-S. (2013). Tunable Resonant-Cavity-Enhanced Photodetector with Double High-Index-Contrast Grating Mirrors. Proceedings of SPIE - The International Society for Optical Engineering, 8633, 86330Y. https://doi.org/10.1117/12.2004047

\section{General rights}

Copyright and moral rights for the publications made accessible in the public portal are retained by the authors and/or other copyright owners and it is a condition of accessing publications that users recognise and abide by the legal requirements associated with these rights.

- Users may download and print one copy of any publication from the public portal for the purpose of private study or research.

- You may not further distribute the material or use it for any profit-making activity or commercial gain

- You may freely distribute the URL identifying the publication in the public portal 


\title{
Tunable Resonant-Cavity-Enhanced Photodetector with Double High- Index-Contrast Grating Mirrors
}

\author{
Supannee Learkthanakhachon, Kresten Yvind, and Il-Sug Chung* \\ Department of Photonics Engineering, Technical University of Denmark, DK-2800 Kgs. Lyngby, \\ Denmark \\ *ilch@fotonik.dtu.dk
}

\begin{abstract}
In this paper, we propose a broadband-tunable resonant-cavity-enhanced photodetector (RCE-PD) structure with double high-index-contrast grating (HCG) mirrors and numerically investigate its characteristics. The detector is designed to operate at 1550-nm wavelength. The detector structure consists of a top InP HCG mirror, a p-i-n photodiode embedding multiple quantum wells, and a Si HCG mirror formed in the Si layer of a silicon-on-insulator wafer. The detection wavelength can be changed by moving the top InP HCG mirror suspended in the air. High reflectivity and small penetration length of HCGs lead to a narrow absorption linewidth of $0.38 \mathrm{~nm}$ and a broad tuning range of $111 \mathrm{~nm}$. The peak absorption efficiency is 76-84\% within the tuning range. This broadband-tunable and narrow-absorption-linewidth RCE-PD is desirable for applications where selective wavelength demultiplexing is required. Furthermore, the fact that it can be fabricated on a silicon platform offers us a possibility of integration with electronics.
\end{abstract}

Keywords: Wavelength division multiplexing, photodiode, HCG, WDM

\section{INTRODUCTION}

Tunable photodetectors with narrow spectral linewidth, large tuning wavelength range, and small footprint are attractive device in applications where capability of wavelength-selective light detection is desirable. For example, in the wavelength division multiplexing-passive optical network (WDM-PON), such a tunable photodetector can be employed in the receiver site of passive broadcasting, i.e., optical network units (ONUs), providing new WDM-PON design possibilities. Portable compact spectrometer is another application of interest.

So far, various tunable photodetector structures have been reported ${ }^{[1]-[9]}$. Among them, the resonant-cavity-enhanced photodetector (RCE-PD) structure is most suitable for a narrow spectral linewidth, thanks to inherent resonance property in RCE-PD ${ }^{[10]}$. As illustrated in Fig. 1, a typical RCE-PD structure consists of two reflectors and an absorption region situated between them. The light absorption in the absorption region is greatly enhanced only at wavelength of resonance in the optical cavity defined by two reflectors. Compared to conventional p-i-n photodiodes, p-i-n RCE-PDs may have higher absorption efficiencies at resonance as well as faster intrinsic response. Absorption efficiency measures the fraction of absorbed light flux over incident light flux. In the beginning research on RCE-PDs, most research efforts have been focused mainly on static wavelength selectivity and related applications, e.g., in ${ }^{\text {[10]-[12] }}$. Then, several approaches for gaining tunable wavelength selectivity have been investigated, using a micromachined movable aluminum mirror ${ }^{[4]}$, the quantum confined Stark effect with an air-semiconductor interface as a mirror ${ }^{[6], ~[7]}$, and a movable distributed Bragg reflector (DBR) ${ }^{[8],[9]}$. To get a narrow spectral linewidth, both reflectors of a RCE-PD need to have a sufficiently high reflectivity. In this respect, a movable DBR approach is ideal. So far, experimentally reported tuning range from the movable DBR approach is $75 \mathrm{~nm}$ with a spectral linewidth of $4 \mathrm{~nm}-6 \mathrm{~nm}{ }^{[9]}$.

In recent years, high-index-contrast subwavelength gratings (HCGs) have received great attention for theirs high, broadband, and polarization-selective reflectivity for normal incident light ${ }^{[13]}$. HCGs, a membrane layer with one- or two-dimensional periodic perforations, are typically made of semiconductor or dielectric material surrounded by low refractive index medium [14], [15]. Due to excellent reflecting characteristics and other novel functionalities, HCGs become an attractive candidate to replace DBRs for an integrated optics e.g. lasers, filters, waveguides, and sensors ${ }^{[13]}$. Recently, we have shown that a tunable vertical-cavity surface-emitting laser (VCSEL), which has a similar cavity structure as RCE-PDs, can have a tuning range as large as $100 \mathrm{~nm}$ by employing a high-index-contrast subwavelength grating (HCG) 
as a movable reflector ${ }^{[16]}$. This motivates the work in this paper. We would like to learn what benefit can be gained by employing HCGs in RCE-PDs.

In this paper, we suggest a tunable RCE-PD structure with double HCGs operating at 1550-nm wavelength. The proposed PD structure is a hybrid structure combining InP material with Si wafer. The movable HCG is made of InP and the other fixed HCG is made of Si. The tuning range, peak absorption efficiency, spectral linewidth, and polarization selectivity are numerically investigated and discussed.

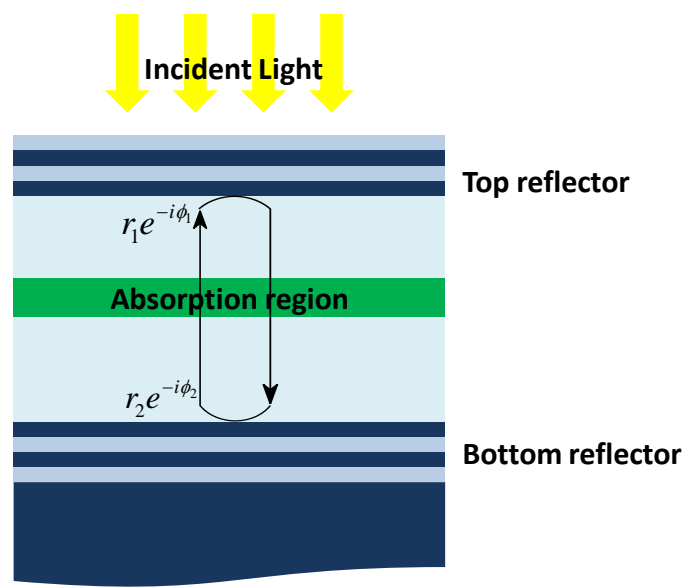

Figure 1. Model of the RCE-PD in which an absorption layer is sandwiched between 2 reflectors.

\section{DEVICE STRUCTURE AND SIMULATION METHOD}

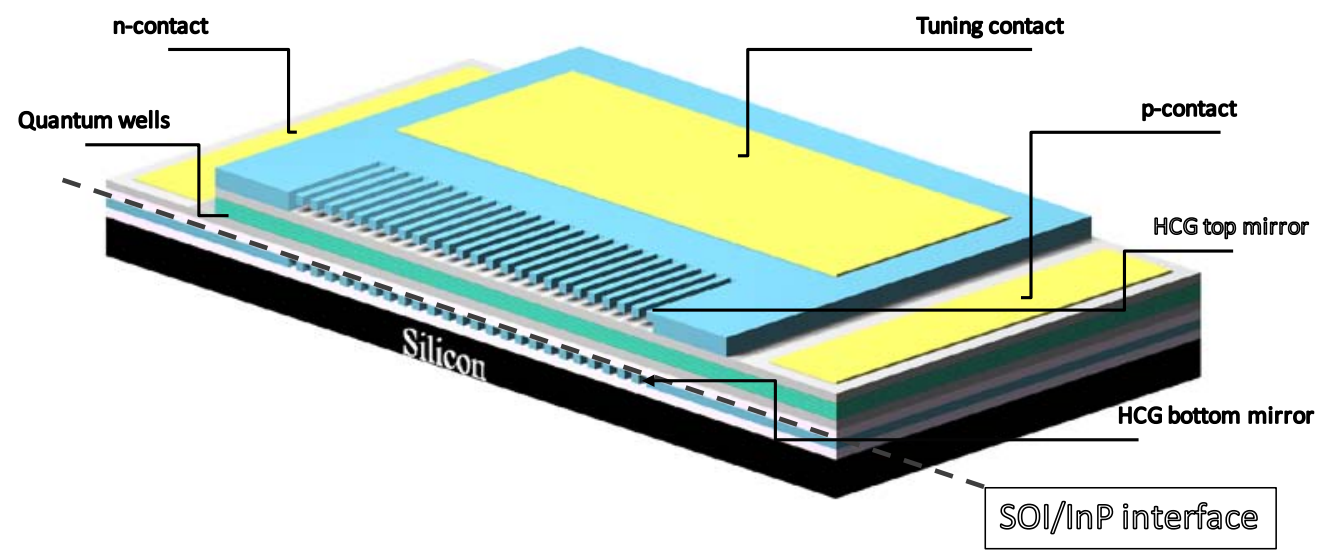

Figure 2. Schematic cross-sectional profile of the proposed tunable RCE-PD with HCGs

As shown in Figure 2, the proposed RCE-PD structure consists of a top n-type InP HCG, a III-V p-i-n diode, and a bottom Si HCG formed in the Si layer of a silicon-on-insulator (SOI) wafer. There is an air gap between the InP HCG and the III-V diode and a SiO2 gap between the III-V diode and the Si HCG, as shown in Figure 3. Both HCGs are surrounded by low refractive index materials. The light is vertically incident to the top InP HCG mirror. By applying a voltage between the tuning contact and the p-contact, the top InP HCG region can be pulled downward, decreasing the thickness of the air gap underneath the InP HCG mirror. In this way, the resonance wavelength, i.e., detection wavelength can be shifted to shorter wavelengths. 


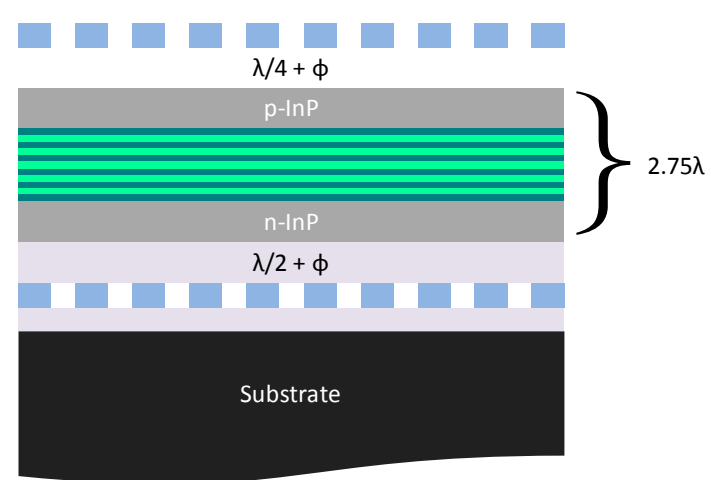

Figure 3. The RCE-PD optical path length design

To maximize the absorption efficiency, the reflectivity of a light incident mirror, $R_{\text {top }}$ should be lower than that of the bottom mirror, $R_{\text {bottom }}$ so that the following relation is satisfied [10]:

$$
R_{\text {top }}=R_{\text {bottom }} e^{-2 \alpha d}
$$

where $\alpha$ is an absorption coefficient and $d$ is the absorption layer thickness, respectively. By numerical simulations with the above relation as the initial guess, the reflectivity values of the top and bottom gratings are designed. The combination of the top InP HCG with $62 \%$ duty cycle, $480-\mathrm{nm}$ thickness and 720 -nm period, and a $\lambda / 4$-thick air gap gives a reflectivity of $99.15 \%$; the thickness of the air gap is designed to produce a destructive interference between reflected light from the HCG and InP/air interface. The combination of the bottom Si HCG with 66\% duty cycle, 500$\mathrm{nm}$ thickness and 730- nm period, and a $\lambda / 2$-thick SiO2 gap gives a reflectivity of $99.92 \%$. The thickness of the SiO2 gap is designed to produce constructive interference. The p-i-n diode embedding five 6.6-nm-thick quantum wells (QWs) is 2.75 $\lambda$-thick. The absorption coefficient of QWs and HCG top mirror are assumed $0.01 \mathrm{~cm}-1$ and $4.31 \times 10^{-5} \mathrm{~cm}-1$, respectively. The refractive indices were 3.1661 for $\mathrm{InP}, 3.4000$ for QWs, 1.4700 for $\mathrm{SiO}_{2}$, and 3.4770 for $\mathrm{Si}$.

To numerically estimate the absorption efficiency, the transfer matrix method (TMM) and the rigorous coupled wave analysis (RCWA) method are combined to calculate the field distribution and Poynting vector. First, the Fresnel reflection and transmission amplitudes of a HCG are calculated by using the RCWA method, and then in the TMM, the HCG is treated as an interface by using the Fresnel amplitudes.

In the TMM method, the absorption coefficient appears in the propagation constant of moving-upward or movingdownward field components, i.e., $E^{+}$or $E^{-}$. However, the amount of absorption is influenced by $\left|E^{+}+E^{-}\right|^{2}$. To correctly consider this standing wave effect in all layers, the effective absorption coefficient of each layer is calculated as follows [17].

$$
\alpha_{\text {eff }}=\frac{\alpha \int_{\text {abs_layers }}\left|E^{+}+E^{-}\right|^{2} d z}{\int \text { abs_layers }\left|E^{+}\right|^{2}+\left|E^{-}\right|^{2} d z}
$$

where $\alpha$ is a material absorption coefficient, $E^{+}\left(E^{-}\right)$is transverse components of the forward(backward) electric field. The integrations are performed within the layer of consideration.

Then, the amount of light absorption in QW layers are calculated by subtracting the out-coming flux from QWs, from the incoming flux into QWs ${ }^{[18]}$.

\section{RESULTS AND DISCUSSION}


Results on the HCG mirror design are presented in Figure 4. The incident electric field is TM-polarized. In this paper, we call polarization perpendicular to the grating grooves as TM polarization, while the parallel one, as TE polarization. Figure 4(a) and 4(b) show the reflectivity and phase of the bottom Si HCG alone are compared with those of the bottom $\mathrm{Si} \mathrm{HCG}$ with a $\mathrm{SiO}_{2}$ gap layer. As shown in Fig. 4(a), the composite reflectivity of the bottom $\mathrm{Si} \mathrm{HCG} \mathrm{with} \mathrm{a} \mathrm{SiO}_{2}$ gap is higher than that of the Si HCG alone, due to the constructive interference. In this way, we can get the target reflectivity of the bottom mirror. The slope of reflection phase is not considerably influenced by this constructive interference, as shown in Fig. 4(b). Knowing this phase slope is important, since it determines the effective phase penetration length into the mirror, $L_{\text {eff }}$;

$$
L_{e f f, i}=\frac{\partial \psi_{i}}{\partial \beta}(i=1,2)
$$

where $\psi$ denotes a phase shift due to light penetrating into the HCG and $\beta=2 n \pi / \lambda$. The $L_{\text {eff }}$ influences the effective total cavity length. On the other hand, as shown in Figure 4(c), the composite reflectivity of the top InP HCG with an air gap layer is lower than the top InP HCG alone, due to destructive interference. As a result, we have shown that it is possible to have a bit lower than $99 \%$ reflectivity by incorporating an air gap spacing to get a destructive interference as can be seen in Figure 4. Using the equation (3), assuming that the reflection phase varies linearly with frequency ${ }^{[16]}$, the effective optical length for the top mirror and bottom mirror was $3.55 \lambda$ and $1.14 \lambda$, respectively. From Eq. (4), the wavelength spacing, called the free spectral range (FSR), between adjacent resonant peaks was $104 \mathrm{~nm}$.

$$
F S R=\frac{\lambda^{2}}{2 n\left(L+L_{e f f, 1}+L_{e f f, 2}\right)}
$$

where $n$ is a refractive index, $L$ is cavity length, and $L_{e f f, i}$ are the effective optical length of the mirrors. The analytical result differs a bit with $111 \mathrm{~nm}$ in the numerical result because the reflection phase of the composite mirrors is not entirely linear.

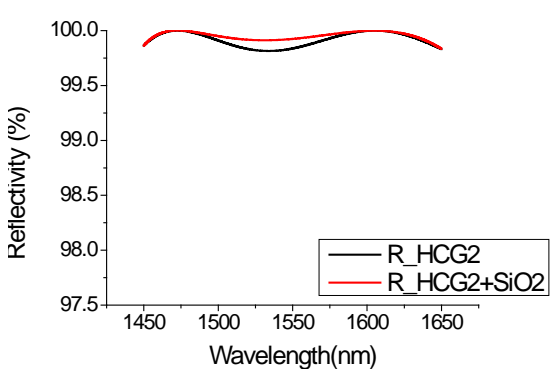

(a)

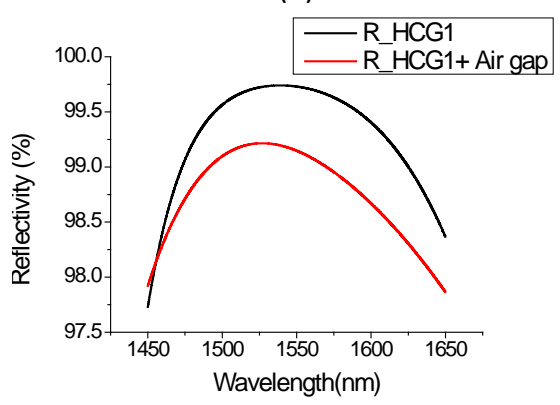

(c)

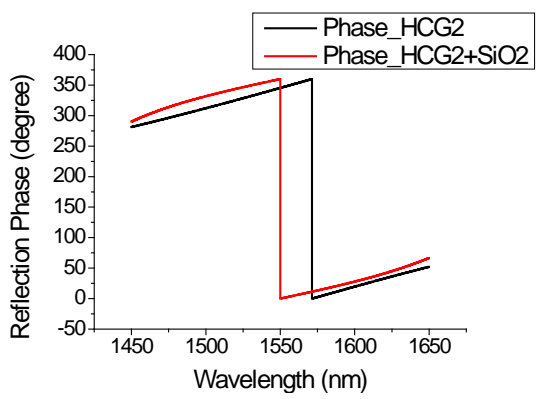

(b)

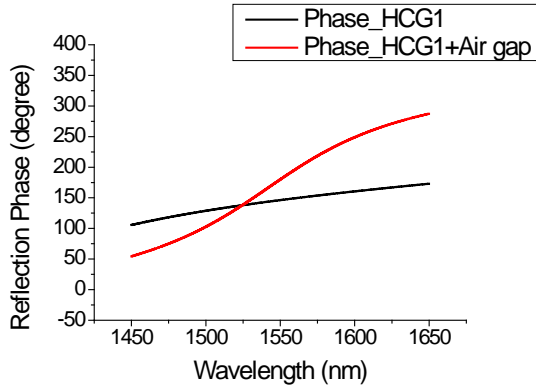

(d)

Figure 4. (a) Reflectivities and (b) reflection phases of the bottom Si HCG alone (black line) and the bottom Si $\mathrm{HCG}+\mathrm{SiO}_{2}$ gap (red line). (c) Reflectivities and (d) reflection phases of the top InP HCG alone (black line) and the top InP HCG + air gap (red line). 
From the numerical simulation, it is possible to have the absorption efficiency stays between $76 \%$ and $84 \%$ across the tuning range. This mean $76 \%$ to $84 \%$ of the input power are absorbed into the quantum well. The rest of the power is lost into other absorbing layers. The variation of the absorption efficiency at different wavelength seen in figure 5 (a) can be explained by changes in effective cavity length which shift the position of the field maxima. These changes cause from physically moving the top mirror and change of phase slope of both mirrors at the off-centred wavelength.

In our RCE-PD structure, a spectral linewidth less $0.38 \mathrm{~nm}$ across the tuning range can be achieved. This value is considerably smaller than the previously reported value of $1.7 \mathrm{~nm}{ }^{[2]}$. Typically, the spectral linewidth of lasers in the WDM system is approximately $0.08 \mathrm{~nm}$ at $10 \mathrm{Gbps}$ modulation speed. Thus, the spectral linewidth of our detector can safely receive the 10 -Gbps modulation signal.

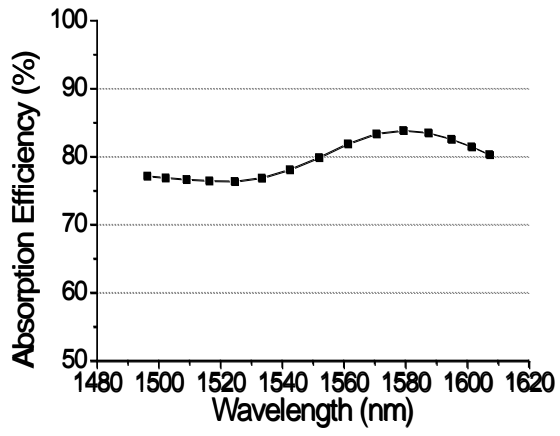

(a)

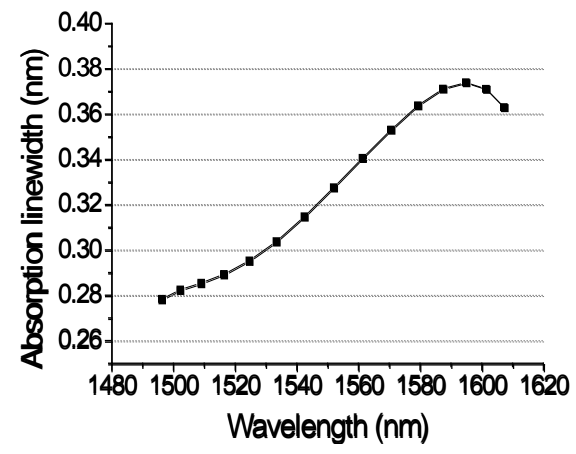

(b)

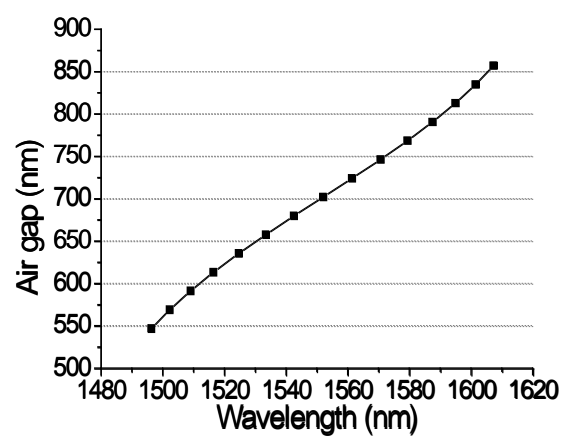

(c)

Figure 5. Tuning result of the RCE-PD (a) absorption efficiency as a function of wavelength (b) absorption linewidth as a function of wavelength and (c) wavelength tuning as a function of air gap spacing.

Light detection in our RCE-PD structures is polarization-selective, thank to the polarization dependence of HCGs. As shown in Figs. 4(c) and 6, the top HCG with an air gap has higher reflectivity for TM-polarized field over tuning wavelength range, compared to the TE-polarized field. Thus, only TM-polarized signal will be detected with high suppression ratio over TE-polarized signal. This property is desirable for polarization-selective applications.

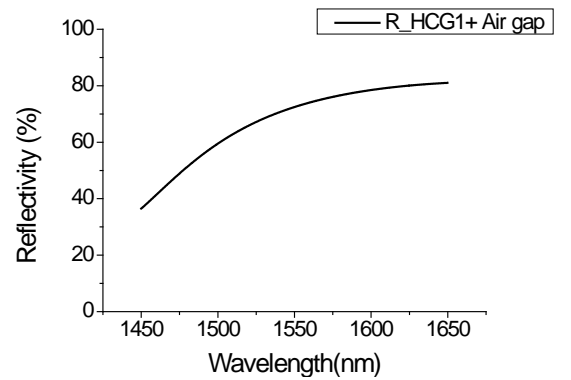

Figure 6. Reflectivity plot from the top composite mirror of TE polarized incident wave. 


\section{CONCLUSION}

We have numerically demonstrated that it is possible to design a tunable RCE-PD with two HCG mirrors so as to provide broad tuning range, high absorption efficiency, and narrow spectral linewidth. The peak absorption efficiency can be as high as around $80 \%$. The tuning wavelength range is $111 \mathrm{~nm}$ which is the same as the FSR of our device. This large FSR is attributed to the small penetration length of HCGs. Thanks to the high reflectivity of HCGs, the spectral absorption linewidth is smaller than $0.38 \mathrm{~nm}$ across the tuning range. The light detection is polarization selective due to polarization-selective reflectivity of HCGs. The hybrid approach proposed can remove the need of GaAs/AlGaAs DBR for RCE-PDs at $1550 \mathrm{~nm}$ wavelength. facilitate the processing in thcombining proposed combination of 2 materials system could enable us an integration of the optoelectronic devices to the silicon platform for more efficient circuit design.

\section{REFERENCES}

[1] T. H. Wood, C. a. Burrus, a. H. Gnauck, J. M. Wiesenfeld, D. a. B. Miller, D. S. Chemla, and T. C. Damen, "Wavelength-selective voltage-tunable photodetector made from multiple quantum wells," Appl. Phys. Lett. 47(3), 190 (1985)

[2] F. Sugihwo, C. Lin, and L. Eyres, "Broadly-tunable narrow-linewidth micromachined laser/photodetector and phototransistor,” Electron Devices Meeting, 1998. IEDM '98. Technical Digest, International, 665-668 (1998).

[3] S. Strite and M. Unlu, "Tunable photodetectors and light-emitting diodes for wavelength division multiplexing,” Electron. Lett. 31(8), 10-12 (1995).

[4] B. Pezeshki and J. S. Harris, "Electrostatically tunable optical device and optical interconnect for processors," US Patent 5,291,502,5 (1994).

[5] R. Chen, D. Miller, and J. S. Harris, "Design and Analysis of CMOS-Controlled Tunable Photodetectors for Multiwavelength Discrimination,” J. Light. Technol. 27(23), 5451-5460 (2009).

[6] K. Lai and J. C. Campbell, "Design of a tunable GaAs/AlGaAs multiple quantum-well resonant-cavity photodetector,” IEEE J. Quant. Electron. 30, 108 (1994).

[7] J. Waclawek and J. Kovac, "Electrically tunable GaAs/AlGaAs MQW RCE photodetector," Electron. Lett. 33(1), $71-72$ (1997).

[8] M. S. Wu, E. C. Vail, G. S. Ly, W. Yuen, and C. J. Chang-Hasnain, "Widely and continuously tunable micromachined resonant cavity detector with wavelength tracking,” IEEE Photon. Technol. Lett. 8, 98 (1996).

[9] G. L. Christenson, a. T. T. D. Tran, Z. H. Zhu, Y. H. Lo, M. Hong, J. P. Mannaerts, and R. Bhat, "Longwavelength resonant vertical-cavity LED/photodetector with a 75-nm tuning range,” IEEE Photon. Technol. Lett. 9(6), 725-727 (1997).

[10] K. Kishino, M. S. Unlu, J.-I. Chyi, J. Reed, L. Arsenault, and H. Morkoc, "Resonant cavity-enhanced (RCE) photodetectors,” IEEE J. Quant. Electron. 27(8), 2025-2034 (1991).

[11]I.-S. Chung, Y. T. Lee, J.-E. Kim, H. Y. Park, "Effect of outermost layers on resonant cavity enhanced devices,” J. Appl., Phys., 96, 2423 (2004).

[12]I.-S. Chung and Y. T. Lee, "A method to tune the cavity-mode wavelength of resonant cavity-enhanced photodetectors for bidirectional optical interconnects,” IEEE Photon. Technol. Lett. 18, 46 (2006).

[13] C. J. Chang-Hasnain, "High-contrast gratings as a new platform for integrated optoelectronics,” Semiconductor Science and Technology 26(1), 014043 (2011).

[14]C. F. R. Mateus, M. C. Y. Huang, L. Chen, C. J. Chang-Hasnain, and Y. Suzuki, "Broad-Band Mirror (1.12$1.62 \mu \mathrm{m})$ Using a Subwavelength Grating,” IEEE Photon. Technol. Lett. 16(7), 1676-1678 (2004).

[15] M. C. Y. Huang, C. Chase, V. Karagodsky, M. Moewe, B. Pesala, F. G. Sedgwick, and C. J. Chang-Hasnain, "High-Index-Contrast Grating (HCG) and Its Applications in Optoelectronic Devices,” IEEE J. Sel. Top. Quant. Electron. 15(5), 1485-1499 (2009). 
[16] I.-S. Chung, V. Iakovlev, A. Sirbu, A. Mereuta, A. Caliman, E. Kapon, and J. Mørk, "Broadband MEMSTunable High-Index-Contrast Subwavelength Grating Long-Wavelength VCSEL,” IEEE J. Quant. Electron. 46(9), 1245-1253, IEEE (2010).

[17] L. A. Coldren and S. W. Corzine, Diode lasers and photonic integrated circuits, Wiley, New York (1995)

[18] Photodetectors and fiber optics, Academic Press, San Diego, CA; London (2001). 